\title{
Comparação de aproximações das equações de Knott- Zoeppritz para modelos com baixo contraste de impedância
}

Victor Jorge de Oliveira Marum* (IPECI/UNISANTOS), Oleg Bokhonok (IPECI/UNISANTOS \& IAG-USP), Liliana Alcazar

Diogo (IAG-USP), Isabella Tomasella Auad (IPECI/UNISANTOS)

Copyright 2016, SBGf - Sociedade Brasileira de Geofísica

Este texto foi preparado para a apresentação no VII Simpósio Brasileiro de Geofísica, Ouro Preto, 25 a 27 de outubro de 2016. Seu conteúdo foi revisado pelo Comitê a mas não necessariamente representa a opinião da SBGf ou de seus associados. É proibida a reprodução total ou parcial deste material para propósitos comerciais sem prévia autorização da SBGf.

\section{Resumo}

A interpretação quantitativa de dados sísmicos usando AVA (Amplitude versus Ângulo) tem se tornado uma técnica importante e bastante utilizada na exploração de hidrocarbonetos. Uma das aplicações dessa técnica consiste em determinar os tipos de rocha e os fluidos contidos em seus poros usando a informação de amplitude de reflexão de ondas sísmicas. O fenômeno de variação de amplitude de onda sísmica com ângulo de incidência é descrito pelas equações exatas de KnottZoeppritz, porém existem aproximações dessas equações que podem servir em determinadas situações e são significativamente mais simples. A importância de investigar os modelos de baixo contraste de impedância existe porque esses modelos estão associados a sinal sísmico de baixa amplitude e, consequentemente, geram pior qualidade da imagem de subsuperfície, tornando-se um grande desafio para interpretação quantitativa. A maioria das aproximações criadas até agora é para situações de baixo contraste de impedância, no entanto, não existem trabalhos que comparam o desempenho destas aproximações para este tipo de modelos. Foram implementadas e comparadas algumas aproximações das equações de Knott-Zoeppritz exatas para ondas planas de reflexão PP (Rpp) e PS (Rps) em modelos geológicos com baixo contraste de impedância. Foi determinado o erro absoluto das curvas do coeficiente de reflexão das aproximações em relação às curvas das equações exatas de Knott-Zoeppritz. O desempenho das aproximações foi comparado para os 3 modelos de baixo contraste de impedância distintos, e pôde-se concluir qual das aproximações testadas apresentou melhor eficiência para cada um destes modelos.

Introdução

A técnica de AVA (Amplitude versus Angle) desempenha um papel importante na interpretação sísmica quantitativa, atuando como indicadora de gás ou hidrocarbonetos no reservatório (Ostrander, 1984). A análise de AVA descreve o comportamento do coeficiente de reflexão (ou refletividade) de uma interface em função do ângulo com que a energia sísmica incide sobre a mesma (Aki \& Richards, 1980). Knott (1899) e Zoeppritz (1919) desenvolveram equações que descrevem o comportamento exato da refletividade de ondas planas compressionais $(P)$ e cisalhantes $(S)$ em uma interface, que é função das densidades e velocidades (de ondas $P$ e S) nas camadas adjacentes. Entretanto, as aproximações das equações exatas de Knott (1899) e Zoeppritz (1919) propostas por alguns autores - como Bortfeld (1961), Aki \& Richards (1980), Shuey (1985), Smith \& Gidlow (1987), Fatti et al. (1994), Gulati \& Stewart (1997), Xu \& Bancroft (1997), Donati \& Martin (1998), Wang (1999), Vant \& Brown (2001) - são umas das mais utilizadas atualmente na indústria de exploração e produção de hidrocarbonetos.

Sabe-se que quanto maior o contraste de impedância entre dois modelos geológicos, maior será o coeficiente de reflexão da interface que os separa e, consequentemente, maior será a amplitude do sinal sísmico gerado na superfície. Quanto maior a amplitude do sinal, melhor é a qualidade da imagem em subsuperfície. Para modelos geológicos com baixo contraste de impedância, a amplitude do sinal tende a ser baixa, o que dificulta a inferência dos parâmetros geológicos do meio. O principal objetivo deste trabalho é comparar as equações exatas de Knott (1899) e Zoeppritz (1919) com as aproximações para ondas PP (Rpp) e PS (Rps), propostas por Aki \& Richards (1980), Fatti et al. (1994) e Wang (1999), para Rpp, e Aki \& Richards (1980), Xu \& Bancroft (1997) e Vant \& Brown (2001), para Rps, e deduzir qual delas gera as curvas dos coeficientes de reflexão com menor erro para o caso dos modelos com baixo contraste de impedância.

\section{Metodologia}

Neste trabalho, as aproximações selecionadas foram aplicadas a três modelos geológicos (Rochas Duras, Rochas Consolidadas e Rochas Inconsolidadas) de baixo contraste de impedância, idealizados no trabalho de Engelmark (2000). Os valores de velocidade de onda $P$ $(V p)$, velocidade de onda $S(V s)$ e densidade $(\rho)$ são de folhelho (camada superior) e de arenito (camada inferior), para cada modelo geológico (Tabela 1).

Aki \& Richards linearizaram os coeficientes de reflexão exatos de Knott-Zoeppritz e chegaram a expressões relativamente simples, para Rpp (Equação 1) e Rps (Equação 2), permitindo com que as variações da amplitude com o afastamento possam ser interpretadas de maneira quantitativa, e é bastante relevante na geofísica de exploração (Ursenbach, 2002).

As aproximações de Rpp e Rps de Aki \& Richards são citadas com frequência por autores que comparam as aproximações para determinados modelos geológicos ou por outros que testam suas próprias aproximações.

$$
R p p=\frac{1}{2 \cos ^{2} \theta} \frac{\Delta \alpha}{\alpha}-2\left(\frac{\beta}{\alpha}\right)^{2} \operatorname{sen}^{2} \theta \frac{\Delta \mu}{\mu}+\frac{1}{2} \frac{\Delta \rho}{\rho}
$$




\begin{tabular}{|c|c|c|}
\hline \multicolumn{3}{|c|}{ Rochas Duras } \\
\hline & Folhelho & Arenito \\
\hline $\mathbf{v p}$ & $4310 \mathrm{~m} / \mathrm{s}$ & $4560 \mathrm{~m} / \mathrm{s}$ \\
\hline Vs & $2552 \mathrm{~m} / \mathrm{s}$ & $3040 \mathrm{~m} / \mathrm{s}$ \\
\hline$\rho$ & $2,55 \mathrm{~g} / \mathrm{cm}^{3}$ & $2,41 \mathrm{~g} / \mathrm{cm}^{3}$ \\
\hline \multicolumn{3}{|c|}{ Rochas Consolidadas } \\
\hline & Folhelho & Arenito \\
\hline$\overline{\mathbf{V p}}$ & $2748 \mathrm{~m} / \mathrm{s}$ & $3048 \mathrm{~m} / \mathrm{s}$ \\
\hline Vs & $1320 \mathrm{~m} / \mathrm{s}$ & $1760 \mathrm{~m} / \mathrm{s}$ \\
\hline$\rho$ & $2,35 \mathrm{~g} / \mathrm{cm}^{3}$ & $2,15 \mathrm{~g} / \mathrm{cm}^{3}$ \\
\hline \multicolumn{3}{|c|}{ Rochas Inconsolidadas } \\
\hline & Folhelho & Arenito \\
\hline$\overline{\mathbf{V p}}$ & $2057 \mathrm{~m} / \mathrm{s}$ & $2134 \mathrm{~m} / \mathrm{s}$ \\
\hline Vs & $490 \mathrm{~m} / \mathrm{s}$ & $889 \mathrm{~m} / \mathrm{s}$ \\
\hline$\rho$ & $2,16 \mathrm{~g} / \mathrm{cm}^{3}$ & $2,08 \mathrm{~g} / \mathrm{cm}^{3}$ \\
\hline
\end{tabular}

Tabela 1 - Parâmetros elásticos de três modelos geológicos com baixo contraste de impedância (Rochas Duras, Rochas Consolidadas e Rochas Inconsolidadas).

$$
\text { Rps }=\frac{-\operatorname{sen} \theta}{2 \cos \varphi}\left\{\frac{\Delta \rho}{\rho}+2\left(\frac{\beta}{\alpha} \cos \theta \cos \varphi-\left(\frac{\beta}{\alpha}\right)^{2} \operatorname{sen}^{2} \theta\right) \frac{\Delta \mu}{\mu}\right\}
$$

Onde,

$$
\frac{\Delta \mu}{\mu} \approx 2 \frac{\Delta \beta}{\beta}+\frac{\Delta \rho}{\rho}
$$

$\mathrm{E} \quad \alpha, \quad \beta, \quad \rho, \Delta \alpha, \quad \Delta \beta, \quad \Delta \rho, \quad \theta$ e $\phi$ representam, respectivamente, a média das velocidades de ondas $P$ das duas camadas, média das velocidades de ondas $S$ das duas camadas, média das densidades das duas camadas, diferença entre as velocidades de ondas $\mathrm{P}$ das duas camadas, diferença entre as velocidades de ondas $\mathrm{S}$ das duas camadas, diferença entre as densidades das duas camadas, média dos ângulos de incidência/reflexão e transmissão de ondas $P$, e média dos ângulos de incidência e transmissão de ondas S.

Fatti et al. (1994) apresentaram uma aproximação em termos de impedância acústica de ondas $\mathrm{P}(\mathrm{I})$ e ondas $\mathrm{S}$ $(J)$, em três termos. O terceiro termo tem peso consideravelmente pequeno para ângulos de incidência inferiores a 35 graus e razão $\alpha / \beta$ entre 1,5 e 2,0. Nesse trabalho foram considerados somente os dois primeiros termos e a aproximação de Fatti et al. (1994), em dois termos, é dada pela Equação 4. Essa aproximação possibilitou a detecção de gás em uma bacia na África do Sul, Mossel Bay, fazendo uso da técnica de Geostack, que é um método que analisa as informações da variação de amplitude sísmica com o afastamento (AVO), dando como resultado uma combinação de traços indicadores de hidrocarbonetos (fator de fluido, $\Delta \mathrm{F}$ ). $\mathrm{O}$ objetivo de incluir a aproximação de Fatti et al. (1994) neste trabalho é verificar se, esta pode ser utilizada na determinação de Rpp para modelos com baixo contraste de impedância.

$$
R p p=\frac{1}{2} \frac{\Delta I}{I}\left(1+\operatorname{tg}^{2} \theta\right)-4\left(\frac{\beta}{\alpha}\right)^{2}\left(\frac{\Delta J}{J}\right) \operatorname{sen}^{2} \theta
$$

Onde I, J, $\Delta l$ e $\Delta J$ representam, respectivamente, a média das impedâncias usando-se ondas $P$ da primeira $e$ segunda camadas, média das impedâncias usando-se ondas $\mathrm{S}$ da primeira e segunda camadas, diferença de impedâncias usando-se ondas $\mathrm{P}$ da primeira e segunda camadas, e diferença de impedâncias usando-se ondas $S$ da primeira e segunda camadas.

Wang (1999) introduziu uma expressão quadrática (Equação 4) e concluiu que esta apresenta maior acurácia quando comparada a sua própria aproximação linear. Sua aproximação foi testada em alguns modelos geológicos, com interfaces entre folhelho/arenito, folhelho/carbonato ou dolomito, anidrita/salmoura $e$ anidrita/carbonato ou dolomito. A aproximação quadrática de Wang foi utilizada neste trabalho para verificar se esta também apresenta um bom ajuste para modelos com baixo contraste de impedância.

$$
\begin{aligned}
R p p= & {\left[\frac{1}{2}-2\left(\frac{\beta}{\alpha}\right)^{2} \operatorname{sen}^{2} \theta\right] \frac{\Delta \rho}{\rho}+\frac{1}{2} \sec ^{2} \theta \frac{\Delta \alpha}{\alpha}+} \\
& -4\left(\frac{\beta}{\alpha}\right)^{2} \operatorname{sen}^{2} \theta \frac{\Delta \beta}{\beta}+ \\
& +\left(\frac{\beta}{\alpha}\right)^{3} \cos \theta \cdot \operatorname{sen}^{2} \theta\left(\frac{\Delta \rho}{\rho}+2 \frac{\Delta \beta}{\beta}\right)^{2}
\end{aligned}
$$

Xu \& Bancroft (1997) derivaram a equação exata de Knott-Zoeppritz e corrigiram a aproximação de Aki \& Richards de Rps, chegando na Equação 5. Os autores fizeram testes em uma sequência geológica de folhelho/arenito e concluíram que sua aproximação apresenta menor erro relativo quando comparada à de Aki \& Richards para esse modelo específico. A aproximação de Xu \& Bancroft foi selecionada com o objetivo de analisar seu comportamento para modelos com baixo contraste de impedância, e também verificar se para este modelo a variação do erro relativo continua menor que a aproximação de Aki \& Richards.

$$
R p s=A\left[P_{o}+P_{1} \cdot \cos (\theta+\varphi)\right]
$$

Onde,

$$
\begin{aligned}
& A=\frac{-\operatorname{sen} \theta}{2 \cos \varphi} \\
& P_{1}=2 \frac{\beta}{\alpha} B
\end{aligned}
$$




$$
\begin{gathered}
B=\frac{\Delta \rho}{\rho}+2 \frac{\Delta \beta}{\beta} \\
P_{o}=\frac{\Delta \rho}{\rho}\left(1+R_{1}\right)-2 \frac{\beta}{\alpha} B \cdot R_{2} \\
R_{1}=\frac{1}{2}\left(\frac{\Delta \rho}{\rho}+\frac{\Delta \beta}{\beta}\right) \\
R_{2}=\frac{1}{2}\left(\frac{\Delta \rho}{\rho}+\frac{\Delta \alpha}{\alpha}\right)
\end{gathered}
$$

Vant \& Brown (2001) também derivaram a equação exata de Knott-Zoeppritz e criaram uma simples aproximação para Rpp (Equação 6), assumindo pequenos ângulos de incidência em vez de pequenas variações dos parâmetros elásticos. A nova aproximação foi testada em três modelos geológicos distintos, sendo dois deles envolvendo parâmetros reversos, isto é, nem todos os parâmetros são alterados na mesma direção da interface, e se mostrou bem ajustada para baixos ângulos de incidência.

$$
R p s=\frac{-2 \theta_{1}\left(\alpha_{2} \beta_{2} \rho_{2} \Delta \rho+2 \rho_{1} \Delta \mu\right)}{\left(\rho_{1} \alpha_{1}+\rho_{2} \alpha_{2}\right)\left(\rho_{1} \beta_{1}+\rho_{2} \beta_{2}\right)}
$$

Onde,

$$
\Delta \mu=\rho_{2} \beta_{2}^{2}-\rho_{1} \beta_{1}^{2}
$$

\section{Resultados}

Nota-se, pela análise da Figura 1 , que as três aproximações testadas para Rpp (Aki \& Richards, 1980, Fatti et al., 1994, e Wang, 1999) mostram-se bem ajustadas até aproximadamente 25 graus para Rochas Duras (A), 20 graus para Rochas Consolidadas (B) e 10 graus para Rochas Inconsolidadas (C). Para os dois primeiros modelos, a aproximação de Aki \& Richards (1980) mostra-se melhor ajustada para ângulos de incidência variando entre 25 e 60 graus. A aproximação de Wang se mostrou melhor ajustada para o terceiro modelo para a mesma faixa de ângulos.

Observa-se pela Figura 2 que, para os três modelos geológicos, as três aproximações testadas para Rps (Aki \& Richards, 1980, Xu \& Bancroft, 1997, e Vant \& Brown, 2001) apresentam-se bem ajustadas até aproximadamente 10 graus. Para Rochas Duras (A) e Rochas Consolidadas (B), as aproximações de Aki \& Richards (1980) e Xu \& Bancroft (1997) mostram-se relativamente bem ajustadas até 25 graus, e para Rochas Inconsolidadas (C) até aproximadamente 20 graus. A aproximação de Vant \& Brown (2001) apresenta o maior erro com o aumento do ângulo de incidência.

A Figura 3 permite uma análise mais detalhada com relação à qualidade do ajuste de cada aproximação para cada tipo de modelo, para os coeficientes de reflexão de ondas PP (A) e PS (B).

\section{Conclusões}

Conclui-se que para a determinação do coeficiente de reflexão de ondas PP (Rpp), a aproximação que apresentou melhor comportamento para os modelos Rochas Duras e Rochas Consolidadas é a de Aki \& Richards (1980). Porém a aproximação de Wang (1999) apresentou menor erro para o modelo de Rochas Inconsolidadas. Para a determinação do coeficiente de reflexão de ondas PS (Rps), a aproximação de Xu \& Bancroft (1997) apresentou melhor comportamento para os modelos Rochas Duras e Rochas Consolidadas, e a aproximação de Aki \& Richards (1980) apresentou menor erro para o modelo de Rochas Inconsolidadas.

Em geral, para a determinação do coeficiente de reflexão de ondas PP a aproximação de Wang (1999) se mostrou mais adequada, e para as ondas PS, o melhor resultado foi obtido com a aproximação de Aki \& Richards (1980).

Pode-se concluir também que as aproximações criadas para calcular o coeficiente de reflexão de onda PS apresentam erro significativamente maior quando comparadas às aproximações para 0 cálculo do coeficiente de reflexão de onda PP. Esse desempenho, um tanto insatisfatório, pode anular as vantagens do uso de ondas PS para análise de AVA, exigindo, então, uma grande necessidade de trabalho intenso para a criação de aproximações mais precisas.

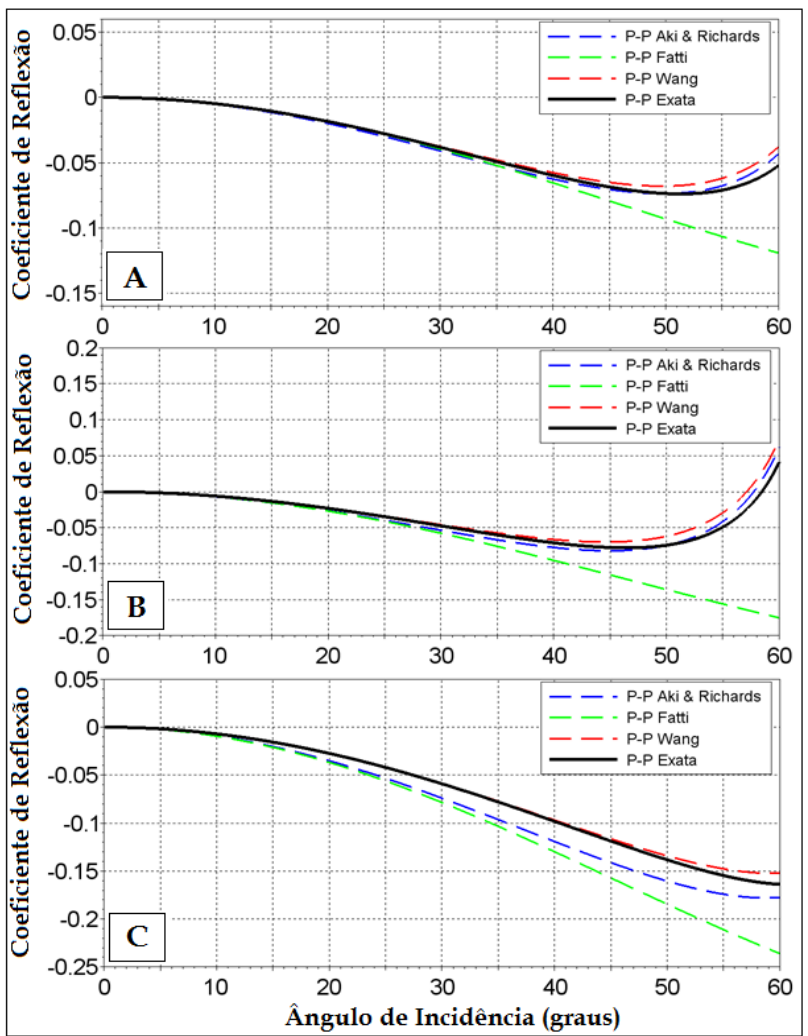

Figura 1 - Comparação das aproximações de Aki \& Richards (1980), Fatti et al. (1994) e Wang (1999) com a equação exata de Zoeppritz, para Rpp, experimentadas em Rochas Duras (A), Rochas Consolidadas (B) e Rochas Inconsolidadas (C). 


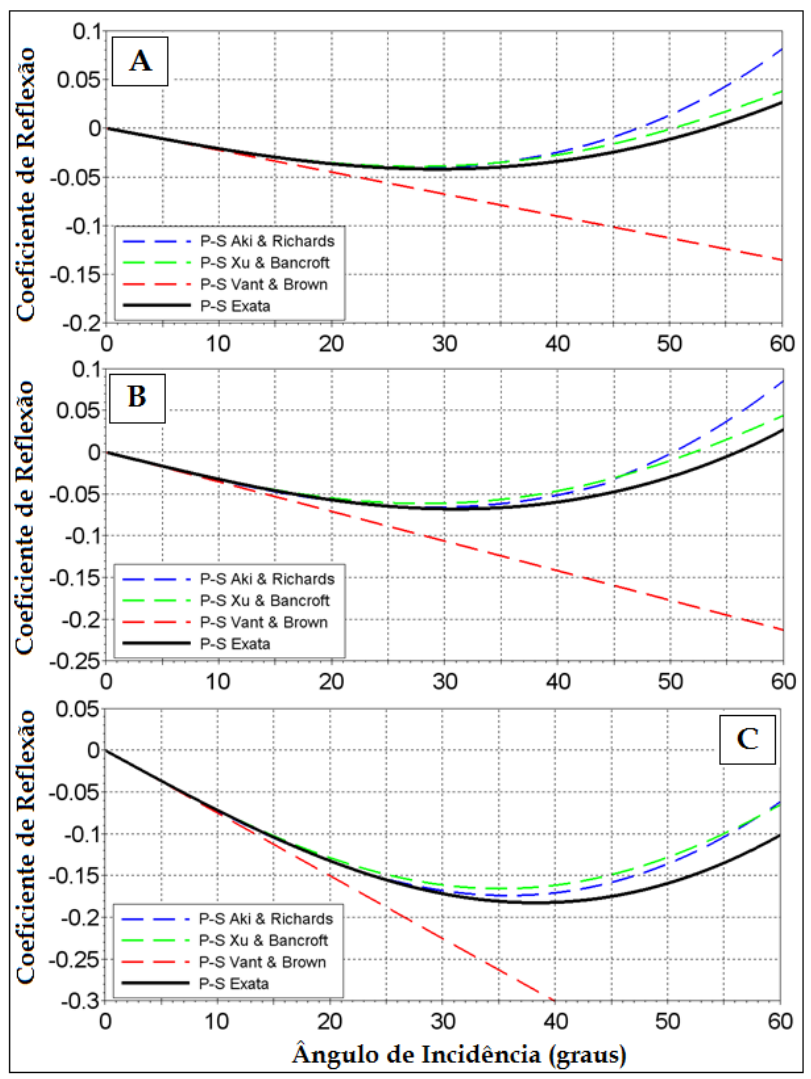

Figura 2 - Comparação das aproximações de Aki \& Richards (1980), Xu \& Bancroft (1997) e Vant \& Brown (2001) com a equação exata de Zoeppritz, para Rps, experimentadas em Rochas Duras (A), Rochas Consolidadas $(B)$ e Rochas Inconsolidadas (C).

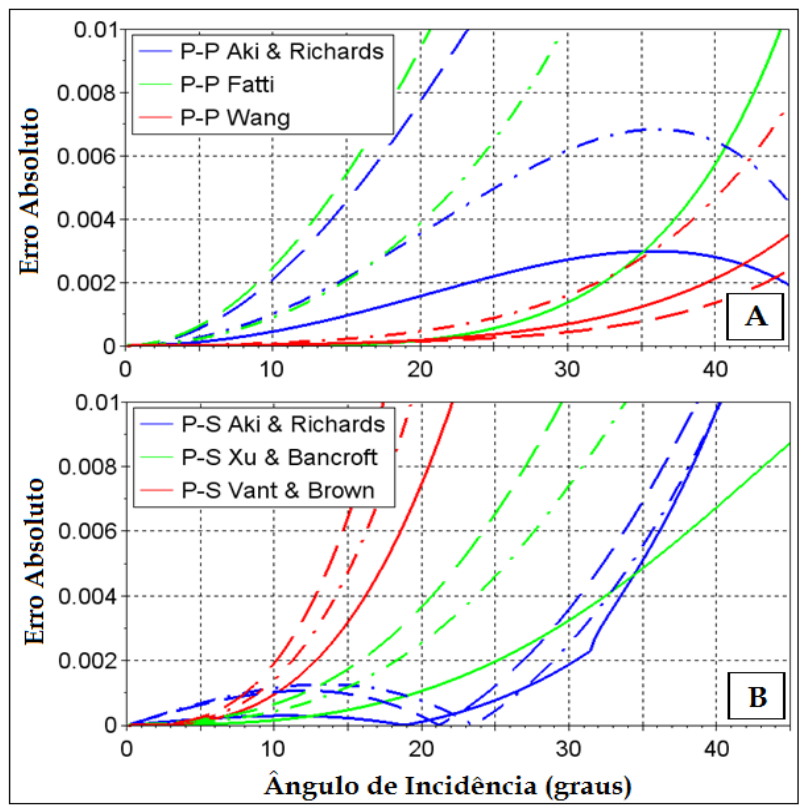

Figura 3 - Comparação da variação do erro absoluto em função do ângulo de incidência para as aproximações de Rpp (A) e Rps (B), (_Rochas Duras; -.- Rochas Consolidadas; e - - - Rochas Inconsolidadas).

\section{Agradecimentos}

VJOM agradece ao Conselho Nacional de Desenvolvimento Científico e Tecnológico (CNPq) pela bolsa de estudos de Iniciação Científica (Processo: 134971/2015-9), à Universidade Católica de Santos, IPECI e a todas as pessoas que nos apoiaram na consecução deste trabalho.

OBC agradece ao Conselho Nacional de Desenvolvimento Científico e Tecnológico (CNPq) pela bolsa de Pós-doutorado (Processo: 159293/2015-4)

\section{Referências}

Aki, K., Richards, P.G., 1980. Quantitative Seismology, Theory and Methods, W. H. Freeman, San Francisco, vol. 1: 119-148.

Bortfeld, R., 1961. Approximations to the reflection and transmission coefficients of plane longitudinal and transverse waves, Geophysical Prospecting, vol. 9: 485502.

Donati, M., Martin, N. W., 1998. A comparison of approximations for the converted-wave reflection, CREWES Research Report, vol. 10.

Engelmark, F., 2000. Using converted shear waves to image reservoirs with low-impedance contrast, The Leading Edge, vol. 19: 600-603.

Fatti, J. L., Smith, G. C., Vail, P. J., Strauss, P. J., Levitt, P. R., 1994. Detection of gas in sandstone reservoirs using AVO analysis: A 3-D seismic case history using the Geostack technique, Geophysics, vol. 59: 1362-1376.

Gulati, J. S., Stewart, R. R., 1997. Analysis of mode conversions over high-velocity layers, CREWES Research Report, vol. 9.

Knott, C. G., 1899. Reflection and refraction of elastic waves, with seismological applications, Phil. Mag, vol. 48: 64-87.

Oladapo, M. I., 2013. Linearization of Zoeppritz equations and practical utilization, International Journal of Physical Sciences, vol. 8: 1298-1306.

Ostrander, W. J., 1984. Plane-wave reflection coefficients for gas sands at nonnormal angles of incidence, Geophysics, vol. 49: 1637-1648.

Shuey, R. T., 1985. A simplification of Zoeppritz equations, Geophysics, vol. 50: 609-614.

Smith, G. C., Gidlow, P. M, 1987. Weighted stacking for rock property estimation and detection of gas, Geophysical Prospecting, vol. 35: 993-1014.

Ursenbach, C. P., 2002. Optimal Zoeppritz approximations, CREWES Research Report, vol. 14: 110.

Vant, A., Brown, R. J., 2001. Approximating the P-S reflection coefficient for small incidence angles, CREWES Research Report, vol. 13: 159-179.

Wang, Y., 1999. Approximations to the Zoeppritz equations and their use in AVO analysis, Geophysics, vol. 64: 1920-1927. 
Xu, Y., Bancroft, J. C., 1997. Joint AVO analysis of PP and PS seismic data, CREWES Research Report, vol. 9.

Zoeppritz, K., 1919. Erdbebenwellen VIII B, Uber Reflexion und durchgang seismischer wellen durch Unstetigkeitsflachen. Gottinger Nachr, vol. 1: 66-84. 